\title{
Solving diophantine equations $x^{4}+y^{4}=q z^{p}$
}

\author{
by \\ Luis V. Dieulefait (Barcelona)
}

1. Introduction. When considering the non-existence of primitive solutions in integers for the equation

$$
x^{4}+y^{4}=q z^{p}
$$

for a fixed prime $q$ and $p$ running over all sufficiently large prime exponents, there are two sets of values of $q$ where the answer is immediate. On the one hand, for primes $q$ that can be written as the sum of two biquadrates, like $17=1+16$, one already has a trivial solution for the equation above for an arbitrary value of $p$ just taking $z=1$. On the other hand, a necessary condition for the existence of primitive solutions in integers is the existence of solutions modulo $q$, and it is easy to see that this is equivalent to the existence of solutions of $u^{4} \equiv-1(\bmod q)$, and since the multiplicative group of the finite field of $q$ elements is cyclic we know that this congruence has solutions if and only if $q \equiv 1(\bmod 8)$. Thus, for any odd prime $q$ not satisfying this condition, (1.1) cannot have primitive solutions, for any value of $p$.

From now on we will restrict to primes $q \equiv 1(\bmod 8)$ such that $q \neq a^{4}+b^{4}$ and we will give an algorithm that, using the modularity of the Q-curve constructed by Darmon-Ellenberg attached to a hypothetical solution of (1.1) and imitating the Frey-Ribet approach, ends up for some values of $q$ in an "impossible congruence", thus proving the non-existence of primitive solutions.

Acknowledgements. I want to thank A. Quirós and F. Holgado for their interesting comments and suggestions on a first version of this article.

2. From non-existence of trivial solutions to non-existence of solutions. Fix a value of $q$ as above (an "interesting" value) and assume that for some $p>13$ there exists a primitive solution $(A, B, C)$ of $(1.1)$. We

2000 Mathematics Subject Classification: 11D41, 11F11.

Research partially supported by MCYT grant BFM2003-01898. 
are assuming in particular that $q$ is odd (because $2=1^{4}+1^{4}$ ), and it is easy to see that $C$ must be odd, and prime to 3 . By assumption, $C \neq 1$. The numbers $A$ and $B$ have different parity, thus we can assume that $A$ is even. Following the construction of Darmon and Ellenberg (cf. [Da], [E]) we can attach, as we did in [Di] for the equation $x^{4}+y^{4}=z^{p}$, two Q-curves to the triple $(A, B, C)$. For simplicity we will just consider one of them (see, however, the remark at the end of this section concerning the case $q=41$ ), namely the curve

$$
E_{(A, B)}: y^{2}=x^{3}+2(1+i) A x^{2}+\left(-B^{2}+i A^{2}\right) x .
$$

It follows from the results in $[\mathrm{E}]$ that $E_{(A, B)}$ is a degree 2 Q-curve defined over $\mathbb{Q}(i)$ semistable outside 2 . Odd primes of bad reduction are precisely the primes dividing $q C$. Thus, the curve has good reduction at 3 , and therefore it is known to be modular (cf. [ES]). Modularity can be interpreted in "Galois language" as follows: consider the compatible family of two-dimensional Galois representations $\varrho_{\lambda}$ of the full Galois group of $\mathbb{Q}$ attached to (the Weil restriction of) $E_{(A, B)}$. There exists a weight 2 cuspidal modular form $f$ such that the representations $\varrho_{\lambda}$ are attached to it, i.e., the trace $a_{t}$ of the image of Frob $t$ agrees with the $t$ th Hecke eigenvalue of $f$, for every prime $t \nmid 2 q C$. We know that the representations $\varrho_{\lambda}$ have coefficients in $\mathbb{Q}(\sqrt{2})$, and moreover that their restrictions to the Galois group of $\mathbb{Q}(i)$ have rational coefficients. Thus, $f$ has an inner twist, i.e., $f^{\sigma}=f \otimes \phi$, where $f^{\sigma}$ is the Galois conjugate of $f$, and $\phi$ is the quadratic character corresponding to $\mathbb{Q}(i)$.

We list some properties of the representations $\varrho_{\lambda}$ that are proved in $[\mathrm{E}]$ : The 2-part of the conductor of this family is 32 (recall that $A$ is even), the residual representations $\bar{\varrho}_{\lambda}$, for every $l>13, \lambda \mid l$, are irreducible, and their (projective) images cannot fall in the normalizer of a split Cartan subgroup of $\mathrm{PGL}_{2}\left(\mathbb{F}_{l}\right)$ (these two properties generalize similar results proved by Mazur and Momose for elliptic curves over $\mathbb{Q}$ ).

The close relation between the discriminant of $E_{(A, B)}$ and $q C^{p}$ is a key point, allowing us to apply Frey's trick: Every odd prime $q^{\prime} \neq q$ of bad reduction of the curve disappears when we consider the (irreducible) residual representation $\varrho_{P}, P \mid p$; this residual representation has conductor $32 q$. Then, applying Ribet's level-lowering result, we conclude that there exists a weight 2 newform $f_{2}$ of level $32 q$ such that, if $\left\{b_{t}\right\}$ is the set of Hecke eigenvalues of $f_{2}$ and $\left\{a_{t}\right\}$ the one of the modular form $f$ associated to $E_{(A, B)}$, we have the congruence

$$
a_{t} \equiv b_{t}(\bmod P)
$$

for every prime $t \nmid 2 q C$.

As $(A, B, C)$ is a primitive solution of (1.1), every prime dividing $C$ is of the form $8 n+1$; in particular, the level of $f$ does not contain any prime $t \equiv 3(\bmod 4)$. 
Now, we want to derive a contradiction from the above mod $P$ congruences by just looking at a few primes $t \equiv 3(\bmod 4)$. On the one hand, we can control the values of the eigenvalues $a_{t}$ of the modular form $f$ (whose level we do not know) for such values of $t$ because $f$ has an inner twist given by the mod 4 character, hence these numbers must satisfy $a_{t}=c \sqrt{2}$, for a rational integer $c$ that we can easily bound using $\left|a_{t}\right| \leq 2 \sqrt{t}$. For example, we know that $a_{3}$ must be one of the following: $0, \pm \sqrt{2}, \pm 2 \sqrt{2}$. On the other hand, $f_{2}$ is new of level $32 q$, so by looking at the tables in [S] (or by applying the Hecke software developed by W. Stein in Magma) we can compute for this finite set of eigenforms the first eigenvalues $b_{t}$.

The desired contradiction will be obtained as long as in the space of modular forms of level $32 q$ no newform has its eigenvalues $b_{t}$ for the first values of $t \equiv 3(\bmod 4)$ of the form $c \sqrt{2}$. If this happens to be the case, then congruences (2.1) will only have solutions for a few primes $p$, and by computing several $b_{t}$ one can shrink this set of primes.

Before computing some examples, we must stress that for certain values of $q$ we know a priori that the method proposed will not be successful. In fact, for a prime $q$ that can be written as a sum of two biquadrates $q=A^{4}+B^{4}$, the method is useless because there will be a newform $f_{2}$ of level $32 q$ whose eigenvalues $b_{t}$ behave precisely as above, namely the newform attached to the Q-curve corresponding to the trivial solution $(A, B, 1)$. In fact, this newform will have field of coefficients $\mathbb{Q}(\sqrt{2})$ and an inner twist by the character corresponding to $\mathbb{Q}(i)$. Thus, a non-trivial solution $\left(A^{\prime}, B^{\prime}, C\right)$ of (1.1) for such values of $q$, that would correspond to a newform $f$ of higher level, may not exist, but we cannot answer this question with our method.

For a similar reason, our method does not work for primes $q$ that can be written as $(2 A)^{4}+B^{2}$ (for example, $41=2^{4}+5^{2}$ ), because even in this case a Q-curve can be attached (cf. [E]) to the triple $(2 A, B, 1)$ and the corresponding newform again will have level $32 q$ and the same field of coefficients and inner twist as above. However, there is still an opportunity to rescue some of these primes: one can consider the second Q-curve attached to a hypothetical solution $\left(A^{\prime}, B^{\prime}, C^{\prime}\right)$ of $x^{4}+y^{4}=q z^{p}$, the curve $E_{\left(B^{\prime}, A^{\prime}\right)}$ defined in [Di] (we are assuming that $B^{\prime}$ is odd). The same method as above can now be applied, this time the congruences relate the eigenvalues of the newform attached to this Q-curve with those of a newform of level $256 q$, and one can hope that in level $256 q$ there is no newform with coefficients in $\mathbb{Q}(\sqrt{2})$ and an inner twist by the mod 4 character (the equation $A^{4}+(2 B)^{2}=$ $q$ has no solutions, thus there is no obvious way to construct a Q-curve such that the corresponding newform has level 256q). This alternative method has not been tested by us in any example because it involves computations with too large levels: $256 q \geq 256 \cdot 41>10000$. 
3. Three successful examples. Let us consider the first three examples of "interesting" primes that cannot be written as $(2 A)^{4}+B^{2}$ :

$$
q=73,89,113 .
$$

We have applied the algorithm described in the previous section to solve the diophantine equations (1.1) for these values of $q$ and $p>13$. To do this, we extracted from the tables in [S] the eigenvalues $b_{3}, b_{7}, b_{11}$ and $b_{19}$ for all newforms of level $32 q$ for these values of $q$. For all the considered newforms at least one of these eigenvalues is not of the form $c \sqrt{2}, c$ an integer. Moreover, solving the congruences $a_{t} \equiv b_{t}(\bmod P)$ where $a_{t}=c \sqrt{2}$, for every integer $c$ such that $\left|a_{t}\right|=|c| \sqrt{2} \leq 2 \sqrt{t}$ and $t=3,7,11$ and 19 , we obtain only one case where these congruences have a solution: $q=73, p=17$ and the newform of level $73 \cdot 32$ named as $2336 L$ in [S] (to solve the congruences, we just compute the primes in the resultant between the minimal polynomials of $a_{t}$ and $b_{t}$ ).

Thus, except for the case $q=73$ and $p=17$, we obtain a contradiction, which proves the following:

ThEOREM 3.1. The diophantine equations $x^{4}+y^{4}=q z^{p}$ do not have primitive solutions for $q=73,89$ and 113 and $p>13$.

REMARK. The method proposed in this article can be used to compute more examples. One can even ask the following question: Is there an interesting prime $q$ (not a sum of two biquadrates) such that the equation $x^{4}+y^{4}=q z^{p}$ has a primitive solution for some $p>13$ ? What if we restrict to primes $q$ not of the form $A^{4}+B^{2}$ ?

It remains to prove the theorem for $q=73$ and $p=17$. We will see that the non-existence of solutions in this case follows from the results in [E].

What happens to the mod 17 representation attached to the newform $2336 L$ is that it has image contained in the normalizer of a Cartan subgroup (in fact, for this modular form, congruences (2.1) hold modulo 17 precisely when we take $a_{t}=0$ for $\left.t \equiv 3(\bmod 4)\right)$. Moreover, this is due to a congruence between $2336 \mathrm{~L}$ and the newform of level 32. The congruence between these two cusp forms can be proved by direct computation, checking that it holds for sufficiently many eigenvalues and applying Sturm's bound. The image is dihedral because of this congruence, since the newform of level 32 has complex multiplication: the Galois representations attached to it are reducible when restricted to the Galois group of $\mathbb{Q}(i)$. As $17 \equiv 1(\bmod 4)$, we see that the mod 17 representation attached to the newform of level 32 has its image contained in the normalizer of a split Cartan subgroup. Now consider the newform $f$ corresponding to a solution of (1.1) with $q=73$ and $p=17$. If we suppose that we have a congruence mod 17 between $f$ and the newform $2336 L$, then we also obtain a congruence between $f$ and 
the newform of level 32, and in particular we conclude that the image of the mod 17 representation attached to $f$ is contained in the normalizer of a split Cartan subgroup; but this contradicts Ellenberg's generalization to Q-curves of results of Momose (see Section 2).

Another way of obtaining a contradiction without applying the results of Ellenberg-Momose is the following: the mod 17 representation attached to the Q-curve cannot have conductor 32, its conductor must be $32 \cdot 73$, because it has semistable reduction at primes above 73 and the formula for the discriminant of this Q-curve shows that the prime 17 does not divide the number of connected components of the reduction of the curve modulo a prime of $\mathbb{Q}(i)$ dividing 73 (the exact power of such a prime dividing the discriminant is not a multiple of 17$)$.

\section{References}

[Da] H. Darmon, Faltings plus epsilon, Wiles plus epsilon, and the generalized Fermat equation, C. R. Math. Rep. Acad. Sci. Canada 19 (1997), 3-14, 64.

[Di] L. Dieulefait, Modular congruences, Q-curves, and the diophantine equation $x^{4}+$ $y^{4}=z^{p}$, Bull. Belgian Math. Soc., to appear.

[E] J. Ellenberg, Galois representations attached to $\mathbb{Q}$-curves and the generalized Fermat equation $A^{4}+B^{2}=C^{p}$, Amer. J. Math. 126 (2004), 763-787.

[ES] J. Ellenberg and C. Skinner, On the modularity of $\mathbb{Q}$-curves, Duke Math. J. 109 (2001), 97-122.

[S] W. Stein, The Modular Forms Database, http://modular.fas.harvard.edu/Tables/.

Dept. d'Algebra i Geometria

Universitat de Barcelona

G. V. de les Corts Catalanes 585

08007 Barcelona, Spain

E-mail: ldieulefait@ub.edu 